\title{
On Certain New Subclass of Meromorphic Close-to-Convex Functions
}

\author{
Jing-Ping Yi, ${ }^{1}$ Zhi-Gang Wang, ${ }^{1}$ and Ming-Hua Zeng ${ }^{2}$ \\ ${ }^{1}$ School of Mathematics and Statistics, Anyang Normal University, Anyang, Henan 455002, China \\ ${ }^{2}$ School of Railway Tracks and Transportation, East China Jiao Tong University, Nanchang, Jiangxi 330013, China \\ Correspondence should be addressed to Zhi-Gang Wang; zhigangwang@foxmail.com
}

Received 19 November 2012; Accepted 31 January 2013

Academic Editor: Mikael Lindstrom

Copyright (C) 2013 Jing-Ping Yi et al. This is an open access article distributed under the Creative Commons Attribution License, which permits unrestricted use, distribution, and reproduction in any medium, provided the original work is properly cited.

We introduce a certain new subclass of meromorphic close-to-convex functions. Such results as inclusion relationship, coefficient inequalities, distortion, and growth theorems for this class of functions are derived.

\section{Introduction}

Let $\Sigma$ denote the class of functions of the form

$$
f(z)=\frac{1}{z}+\sum_{n=1}^{\infty} a_{n} z^{n}
$$

which are analytic in the punctured open unit disk:

$$
\mathbb{U}^{*}:=\{z: z \in \mathbb{C} \text { and } 0<|z|<1\}:=\mathbb{U} \backslash\{0\}
$$

Let $\mathscr{P}$ denote the class of functions $p$ given by

$$
p(z)=1+\sum_{n=1}^{\infty} p_{n} z^{n} \quad(z \in \mathbb{U})
$$

which are analytic and convex in $\mathbb{U}$ and satisfy the following condition:

$$
\Re(p(z))>0 \quad(z \in \mathbb{U}) .
$$

A function $f \in \Sigma$ is said to be in the class $\mathscr{M} \mathcal{S}^{*}(\alpha)$ of meromorphic starlike functions of order $\alpha$ if it satisfies the following inequality:

$$
\mathfrak{R}\left(-\frac{z f^{\prime}(z)}{f(z)}\right)>\alpha \quad(0 \leqq \alpha<1 ; z \in \mathbb{U}) .
$$

Moreover, a function $f \in \Sigma$ is said to be in the class $\mathscr{M C}$ of meromorphic close-to-convex functions if it satisfies the following condition:

$$
\mathfrak{R}\left(-\frac{z f^{\prime}(z)}{g(z)}\right)>0 \quad\left(z \in \mathbb{U} ; g \in \mathscr{M} \mathcal{S}^{*}(0)=: \mathscr{M} \mathcal{S}^{*}\right) \text {. }
$$

For two functions $f$ and $g$ analytic in $\mathbb{U}$, we say that the function $f(z)$ is subordinate to $g(z)$ in $\mathbb{U}$ and write $f(z) \prec$ $g(z)(z \in \mathbb{U})$, if there exists a Schwarz function $\omega(z)$, analytic in $\mathbb{U}$ with $\omega(0)=0$ and $|\omega(z)|<1$ such that $f(z)=g(\omega(z))$. Indeed, it is well known that

$$
\begin{gathered}
f(z) \prec g(z)(z \in \mathbb{U}) \Longrightarrow f(0)=g(0), \\
f(\mathbb{U}) \subset g(\mathbb{U}) .
\end{gathered}
$$

Furthermore, if the function $g$ is univalent in $\mathbb{U}$, then we have the following equivalence:

$$
\begin{gathered}
f(z) \prec g(z)(z \in \mathbb{U}) \Longleftrightarrow f(0)=g(0), \\
f(\mathbb{U}) \subset g(\mathbb{U}) .
\end{gathered}
$$

Recently, Wang et al. [1] introduced and investigated the class $\mathscr{M} \mathscr{K}$ of meromorphic close-to-convex functions which satisfy the inequality

$$
\Re\left(\frac{f^{\prime}(z)}{g(z) g(-z)}\right)>0 \quad(z \in \mathbb{U}),
$$


where $g \in \mathscr{M} \delta^{*}(1 / 2)$. Kowalczyk and Leś-Bomba [2] discussed the class $\mathscr{K}_{s}(\gamma)$ of analytic functions related to the starlike functions; a function $f(z)=z+\sum_{n=2}^{\infty} a_{n} z^{n}$ which is analytic in $\mathbb{U}$ is said to be in the class $\mathscr{K}_{s}(\gamma)(0 \leqq \gamma<1)$, if it is satisfies the following inequality:

$$
\mathfrak{R}\left(\frac{z^{2} f^{\prime}(z)}{g(z) g(-z)}\right)>\gamma \quad(z \in \mathbb{U}),
$$

where $g \in \mathcal{S}^{*}(1 / 2)$. Şeker [3] discussed the class $\mathscr{K}_{s}^{(k)}(\gamma)$ of analytic functions which satisfy the following condition:

$$
\Re\left(\frac{z^{k} f^{\prime}(z)}{g_{k}(z)}\right)>\gamma \quad(z \in \mathbb{U}),
$$

where $0 \leqq \gamma<1, g_{k}(z)=\prod_{v=0}^{k-1} \varepsilon^{-v} g\left(\varepsilon^{v} z\right)\left(\varepsilon^{k}=1 ; k \geqq 1\right)$, and $g \in \mathcal{S}^{*}((k-1) / k)$.

Motivated essentially by the classes $\mathscr{M} \mathscr{K}, \mathscr{K}_{s}(\gamma)$, and $\mathscr{K}_{s}^{(k)}(\gamma)$, we introduce and study the following more generalized class $\mathscr{M} \mathscr{K}^{(k)}(\gamma)$ of meromorphic functions.

Definition 1. A function $f \in \Sigma$ is said to be in the class $\mathscr{M} \mathscr{K}^{(k)}(\gamma)$ if it satisfies the following inequality:

$$
\mathfrak{R}\left(-\frac{f^{\prime}(z)}{z^{k-2} g_{k}(z)}\right)>\gamma \quad(z \in \mathbb{U}),
$$

where $0 \leqq \gamma<1, k \geqq 1$ is a fixed positive integer, and $g_{k}(z)$ is given by

$$
g_{k}(z)=\prod_{v=0}^{k-1} \varepsilon^{v} g\left(\varepsilon^{v} z\right) \quad\left(\varepsilon=e^{(2 \pi i) / k} ; g \in \mathscr{M} \mathcal{S}^{*}\left(\frac{k-1}{k}\right)\right) .
$$

We observe that the inequality (12) is equivalent to

$$
\left|\frac{f^{\prime}(z)}{z^{k-2} g_{k}(z)}+1\right|<\left|\frac{f^{\prime}(z)}{z^{k-2} g_{k}(z)}+2 \gamma-1\right| \quad(z \in \mathbb{U}) .
$$

Since $\mathscr{M} \mathscr{K}^{(2)}(0)=\mathscr{M} \mathscr{K}$, the class $\mathscr{M} \mathscr{K}^{(k)}(\gamma)$ is a generalization of the class $\mathscr{M} \mathscr{K}$.

For some recent investigations on the class of closeto-convex functions, one can find them in [4-7] and the references cited therein. In the present paper, we aim at proving that the class $\mathscr{M} \mathscr{K}^{(k)}(\gamma)$ is a subclass of meromorphic close-to-convex functions. Furthermore, some interesting results of the class $\mathscr{M} \mathscr{K}^{(k)}(\gamma)$ are derived.

\section{Preliminary Results}

To prove our main results, we need the following lemmas.

Lemma 2. Let $\varphi_{j} \in \mathscr{M} \delta^{*}\left(\alpha_{j}\right)$, where $0 \leqq \alpha_{j}<1(j=$ $0,1, \ldots, k-1)$. Then for $k-1 \leqq \sum_{j=0}^{k-1} \alpha_{j}<k$, one has

$$
z^{k-1} \prod_{j=0}^{k-1} \varphi_{j}(z) \in \mathscr{M} \mathcal{S}^{*}\left(\sum_{j=0}^{k-1} \alpha_{j}-(k-1)\right) .
$$

Proof. Since $\varphi_{j} \in \mathscr{M} \mathcal{S}^{*}\left(\alpha_{j}\right)(j=0,1, \ldots, k-1)$, we have

$$
\begin{gathered}
\mathfrak{R}\left(-\frac{z \varphi_{0}^{\prime}(z)}{\varphi_{0}(z)}\right)>\alpha_{0}, \\
\mathfrak{R}\left(-\frac{z \varphi_{1}^{\prime}(z)}{\varphi_{1}(z)}\right)>\alpha_{1}, \ldots, \mathfrak{R}\left(-\frac{z \varphi_{k-1}^{\prime}(z)}{\varphi_{k-1}(z)}\right)>\alpha_{k-1} .
\end{gathered}
$$

We now let

$$
F(z)=z^{k-1} \varphi_{0}(z) \varphi_{1}(z) \cdots \varphi_{k-1}(z) .
$$

Differentiating (17) logarithmically, we obtain

$$
\begin{aligned}
-\frac{z F^{\prime}(z)}{F(z)}= & -\frac{z \varphi_{0}^{\prime}(z)}{\varphi_{0}(z)}-\frac{z \varphi_{1}^{\prime}(z)}{\varphi_{1}(z)} \\
& -\cdots-\frac{z \varphi_{k-1}^{\prime}(z)}{\varphi_{k-1}(z)}-(k-1) .
\end{aligned}
$$

From (18) together with (16), we can get

$$
\begin{aligned}
\Re\left(-\frac{z F^{\prime}(z)}{F(z)}\right)= & \Re\left(-\frac{z \varphi_{0}^{\prime}(z)}{\varphi_{0}(z)}\right)+\mathfrak{R}\left(-\frac{z \varphi_{1}^{\prime}(z)}{\varphi_{1}(z)}\right) \\
& +\cdots+\mathfrak{R}\left(-\frac{z \varphi_{k-1}^{\prime}(z)}{\varphi_{k-1}(z)}\right)-(k-1) \\
> & \sum_{j=0}^{k-1} \alpha_{j}-(k-1) .
\end{aligned}
$$

Thus, if $0 \leqq \sum_{j=0}^{k-1} \alpha_{j}-(k-1)<1$, we know that

$$
F(z)=z^{k-1} \prod_{j=0}^{k-1} \varphi_{j}(z) \in \mathscr{M} \mathcal{S}^{*}\left(\sum_{j=0}^{k-1} \alpha_{j}-(k-1)\right) .
$$

Lemma 3 (see [8]). Let $-1 \leqq B_{2} \leqq B_{1}<A_{1} \leqq A_{2} \leqq 1$. Then

$$
\frac{1+A_{1} z}{1+B_{1} z} \prec \frac{1+A_{2} z}{1+B_{2} z} \text {. }
$$

Lemma 4 (see [9]). Suppose that $g \in M \delta^{*}$. Then

$$
\frac{(1-r)^{2}}{r} \leqq|g(z)| \leqq \frac{(1+r)^{2}}{r} \quad(|z|=r ; 0<r<1) .
$$

Lemma 5 (see [10, page 105]). If the function

$$
p(z)=1+\sum_{n=1}^{\infty} p_{n} z^{n} \quad(z \in \mathbb{U})
$$

analytic and convex in $\mathbb{U}$ and satisfies the condition

$$
\mathfrak{R}(p(z))>\gamma \quad(z \in \mathbb{U}),
$$

then

$$
\frac{1-(1-2 \gamma) r}{1+r} \leqq|p(z)| \leqq \frac{1+(1-2 \gamma) r}{1-r} \quad(|z|=r<1) .
$$


Lemma 6 (see [10]). If the function $p \in \mathscr{P}$ is given by (3), then

$$
\left|p_{n}\right| \leqq 2 \quad(n \in \mathbb{N}) \text {, }
$$$$
\frac{1-r}{1+r} \leqq|p(z)| \leqq \frac{1+r}{1-r} \quad(|z|=r ; 0<r<1) .
$$

Lemma 7 (see [11]). Suppose that

$$
g(z)=\frac{1}{z}+\sum_{n=1}^{\infty} c_{n} z^{n} \in \mathscr{M} \mathcal{S}^{*} .
$$

Then

$$
\left|c_{n}\right| \leqq \frac{2}{n+1} \quad(n \in \mathbb{N}:=\{1,2, \ldots\}) .
$$

Each of these inequalities is sharp, with the extremal function given by

$$
g(z)=z^{-1}\left(1+z^{n+1}\right)^{2 /(n+1)}
$$

Lemma 8 (see [12]). Let

$$
f(z)=1+\sum_{n=1}^{\infty} c_{n} z^{n}
$$

be analytic in $\mathbb{U}$ and let

$$
g(z)=1+\sum_{n=1}^{\infty} d_{n} z^{n}
$$

be analytic and convex in $\mathbb{U}$. If $f \prec g$, then

$$
\left|c_{n}\right| \leqq\left|d_{1}\right| \quad(n \in \mathbb{N}:=\{1,2, \ldots\}) .
$$

Lemma 9. If $p(z) \prec(1+(1-2 \gamma) z) /(1-z)$, where $p(z)$ is given by (3), then

$$
\left|p_{n}\right| \leqq 2(1-\gamma) \text {. }
$$

Proof. By Lemma 8, we easily get the assertion of Lemma 9.

\section{Main Results}

We first give the following result.

Theorem 10. Let $g(z)=1 / z+\sum_{n=1}^{\infty} b_{n} z^{n} \in \mathscr{M}^{*}((k-1) / k)$, Then

$$
G_{k}(z)=z^{k-1} g_{k}(z)=\frac{1}{z}+\sum_{n=1}^{\infty} B_{n} z^{n} \in \mathscr{M} \mathcal{S}^{*},
$$

where $g_{k}(z)$ is given by (13).

Proof. From (13), we know that

$$
\begin{aligned}
z^{k-1} g_{k}(z) & =z^{k-1} \prod_{v=0}^{k-1} \varepsilon^{v} g\left(\varepsilon^{v} z\right) \\
& =z^{k-1} \prod_{v=0}^{k-1} \varepsilon^{v}\left(\frac{1}{\varepsilon^{v} z}+\sum_{n=1}^{\infty} b_{n}\left(\varepsilon^{v} z\right)^{n}\right) \\
& =z^{k-1} \prod_{v=0}^{k-1}\left(\frac{1}{z}+\sum_{n=1}^{\infty} b_{n} \varepsilon^{(n+1) v} z^{n}\right) .
\end{aligned}
$$

Now, suppose that

$$
g(z)=\frac{1}{z}+\sum_{n=1}^{\infty} b_{n} z^{n} \in M \mathcal{S}^{*}\left(\frac{k-1}{k}\right) .
$$

Then, by Lemma 2 and (35), we get the assertion of Theorem 10 easily.

Remark 11. From Theorem 10 and Definition 1, we know that if $f \in \mathscr{M} \mathscr{K}^{k}(\gamma)$, then $f$ is a meromorphic close-to-convex function. So the class $\mathscr{M} \mathscr{K}^{k}(\gamma)$ is a subclass of meromorphic close-to-convex functions.

Now, we prove a sufficient condition for functions to belong to the class $\mathscr{M} \mathscr{K}^{k}(\gamma)$.

Theorem 12. Let $f \in \Sigma, g \in M \mathcal{S}^{*}((k-1) / k)$. Iffor $0 \leqq \gamma<1$, one has

$$
2 \sum_{n=1}^{\infty} n\left|a_{n}\right|+(|1-2 \gamma|+1) \sum_{n=1}^{\infty}\left|B_{n}\right| \leqq 2(1-\gamma),
$$

where the coefficients $B_{n}(n=1,2, \ldots)$ are given by (34), then $f \in \mathscr{M} \mathscr{K}^{k}(\gamma)$.

Proof. We set for $f$ given by (1) and $g_{k}$ defined by (13)

$$
\begin{aligned}
\Delta= & \left|-z f^{\prime}(z)-z^{k-1} g_{k}(z)\right| \\
& -\left|-z f^{\prime}(z)+(1-2 \gamma) z^{k-1} g_{k}(z)\right| \\
= & \left|-\sum_{n=1}^{\infty} n a_{n} z^{n}-\sum_{n=1}^{\infty} B_{n} z^{n}\right| \\
& -\left|\frac{2-2 \gamma}{z}-\sum_{n=1}^{\infty} n a_{n} z^{n}+(1-2 \gamma) \sum_{n=1}^{\infty} B_{n} z^{n}\right| .
\end{aligned}
$$

For $|z|=r(0<r<1)$, from inequality (37), we have

$$
\begin{aligned}
\Delta \leqq & \sum_{n=1}^{\infty} n\left|a_{n}\right||z|^{n}+\sum_{n=1}^{\infty}\left|B_{n}\right||z|^{n} \\
& -\left((2-2 \gamma) \frac{1}{|z|}-\sum_{n=1}^{\infty} n\left|a_{n}\right||z|^{n}-|1-2 \gamma| \sum_{n=1}^{\infty}\left|B_{n}\right||z|^{n}\right) \\
= & -(2-2 \gamma) \frac{1}{|z|}+2 \sum_{n=1}^{\infty} n\left|a_{n}\right||z|^{n}+(|1-2 \gamma|+1) \\
& \times \sum_{n=1}^{\infty}\left|B_{n}\right||z|^{n} \\
< & \left(-(2-2 \gamma)+2 \sum_{n=1}^{\infty} n\left|a_{n}\right|+(|1-2 \gamma|+1) \sum_{n=1}^{\infty}\left|B_{n}\right|\right) \frac{1}{|z|} \\
\leqq & 0 .
\end{aligned}
$$


Thus, we have

$$
\left|-\frac{f^{\prime}(z)}{z^{k-2} g_{k}(z)}-1\right|<\left|-\frac{f^{\prime}(z)}{z^{k-2} g_{k}(z)}+1-2 \gamma\right| \quad(z \in \mathbb{U}),
$$

that is, $f \in \mathscr{M} \mathscr{K}^{(k)}(\gamma)$. This completes the proof of Theorem 12.

Next, we give the inclusion relationship for class $f \in$ $\mathscr{M} \mathscr{K}^{(k)}(\gamma)$.

Theorem 13. Let $0 \leqq \gamma_{1} \leqq \gamma_{2}<1$. Then one has

$$
\mathscr{M} \mathscr{K}^{(k)}\left(\gamma_{2}\right) \subset \mathscr{M} \mathscr{K}^{(k)}\left(\gamma_{1}\right) .
$$

Proof. Suppose that $f \in \mathscr{M}_{\mathscr{K}^{(k)}}\left(\gamma_{2}\right)$. By Definition 1, we have

$$
-\frac{f^{\prime}(z)}{z^{k-2} g_{k}(z)} \prec \frac{1+\left(1-2 \gamma_{2}\right) z}{1-z} .
$$

Since $0 \leqq \gamma_{1} \leqq \gamma_{2}<1$, we get

$$
-1<1-2 \gamma_{2} \leqq 1-2 \gamma_{1} \leqq 1 .
$$

Thus, by Lemma 3, we obtain

$$
-\frac{f^{\prime}(z)}{z^{k-2} g_{k}(z)} \prec \frac{1+\left(1-2 \gamma_{1}\right) z}{1-z},
$$

that is, $f \in \mathscr{M} \mathscr{K}^{(k)}\left(\gamma_{1}\right)$. This means that $\mathscr{M} \mathscr{K}^{(k)}\left(\gamma_{2}\right) \subset$ $\mathscr{M} \mathscr{K}^{(k)}\left(\gamma_{1}\right)$. Hence the proof is completed.

In what follows, we derive the coefficient inequality for the class $\mathscr{M} \mathscr{K}^{(k)}(\gamma)$.

Theorem 14. Suppose that

$$
f(z)=\frac{1}{z}+\sum_{n=1}^{\infty} a_{n} z^{n} \in \mathscr{M} \mathscr{K}^{(k)}(\gamma) .
$$

Then

$$
\left|a_{1}\right| \leqq 1
$$

$$
\begin{aligned}
\left|a_{n}\right| \leqq & \frac{2}{n(n+1)} \\
& +\frac{(2-2 \gamma)}{n}\left(1+\sum_{j=1}^{n-1} \frac{2}{j+1}\right) \quad(n \in \mathbb{N} \backslash\{1\}) .
\end{aligned}
$$

Proof. Suppose that $f \in \mathscr{M} \mathscr{K}^{(k)}(\gamma)$; we know that

$$
\mathfrak{R}\left(-\frac{z f^{\prime}(z)}{G_{k}(z)}\right)>\gamma,
$$

where $G_{k}$ is given by (34). If we set

$$
q(z):=-\frac{z f^{\prime}(z)}{G_{k}(z)}
$$

it follows that

$$
q(z)=1+\sum_{n=1}^{\infty} d_{n} z^{n}
$$

In view of Definition 1 and Lemma 9, we know that

$$
\left|d_{n}\right| \leqq(2-2 \gamma) \quad(n \in \mathbb{N}) .
$$

By substituting the series expressions of functions $f, G_{k}$, and $q$ into (49), we obtain

$$
\left(1+\sum_{n=1}^{\infty} d_{n} z^{n}\right)\left(\frac{1}{z}+\sum_{n=1}^{\infty} B_{n} z^{n}\right)=\left(\frac{1}{z}-\sum_{n=1}^{\infty} n a_{n} z^{n}\right) .
$$

Since $f$ is univalent in $\mathbb{U}^{*}$, it is well known that

$$
\left|a_{1}\right| \leqq 1 \text {. }
$$

On the other hand, we find from (52) that

$$
-n a_{n}=B_{n}+d_{n+1}+\sum_{j=1}^{n-1} B_{j} d_{n-j} \quad(n \in \mathbb{N}) .
$$

Combining (28), (51), and (54), we have

$$
n\left|a_{n}\right| \leqq \frac{2}{n+1}+(2-2 \gamma)+(2-2 \gamma) \sum_{j=1}^{n-1} \frac{2}{j+1} .
$$

Thus, the assertion (47) of Theorem 14 follows directly from (55).

Finally, we give the distortion and growth theorems for the function class $\mathscr{M} \mathscr{K}^{(k)}(\gamma)$.

Theorem 15. If $f \in \mathscr{M} \mathscr{K}^{(k)}(\gamma)$, then

$$
\begin{aligned}
& \frac{(1-r)^{2}[1-(1-2 \gamma) r]}{r^{2}(1+r)} \leqq\left|f^{\prime}(z)\right| \\
& \leqq \frac{(1+r)^{2}[1+(1-2 \gamma) r]}{r^{2}(1-r)} \\
& \quad(|z|=r<1),
\end{aligned}
$$

$$
\begin{aligned}
\int_{0}^{r} \frac{(1-t)^{2}[1-(1-2 \gamma) t]}{t^{2}(1+t)} d t & \leqq|f(z)| \\
& \leqq \int_{0}^{r} \frac{(1+t)^{2}[1+(1-2 \gamma) t]}{t^{2}(1-t)} d t
\end{aligned}
$$

Proof. If $f \in \mathscr{M} \mathscr{K}^{(k)}(\gamma)$, then there exists a function $g \in \mathscr{M} \mathcal{S}^{*}((k-1) / k)$ such that (12) holds. It follows from Theorem 10 that the function given by (34) is a meromorphic starlike function. Therefore, by Lemma 4 , we have

$$
\frac{(1-r)^{2}}{r} \leqq\left|G_{k}(z)\right| \leqq \frac{(1+r)^{2}}{r} \quad(|z|=r ; 0<r<1) .
$$


Let $q$ be defined by (49); by Lemma 5, we know that

$$
\frac{1-(1-2 \gamma) r}{1+r} \leqq|q(z)| \leqq \frac{1+(1-2 \gamma) r}{1-r} \quad(|z|=r<1) .
$$

Thus, from (49), (58), and (59), we readily get (56). Upon integrating (56) from 0 to $r$, we get (57). The proof of Theorem 15 is thus completed.

\section{Acknowledgments}

The present investigation was supported by the National Natural Science Foundation under Grant 11226088, the Open Fund Project of Key Research Institute of Philosophies and Social Sciences in Hunan Universities under Grants 11FEFM02 and 12FEFM02, the Key Project of Natural Science Foundation of Educational Committee of Henan Province under Grant 12A110002, and the Science and Technology Program of Educational Department of Jiangxi Province under Grant GJJ12322 of China. The authors are grateful to the referees for their valuable comments and suggestions which essentially improved the quality of the paper.

\section{References}

[1] Z.-G. Wang, Y. Sun, and N. Xu, "Some properties of certain meromorphic close-to-convex functions," Applied Mathematics Letters, vol. 25, no. 3, pp. 454-460, 2012.

[2] J. Kowalczyk and E. Leś-Bomba, "On a subclass of close-to-convex functions," Applied Mathematics Letters, vol. 23, no. 10, pp. 1147-1151, 2010.

[3] B. Şeker, "On certain new subclass of close-to-convex functions," Applied Mathematics and Computation, vol. 218, no. 3, pp. 1041-1045, 2011.

[4] P. Goswami, S. Bulut, and T. Bulboacă, "Certain properties of a new subclass of close-to-convex functions," Arabian Journal of Mathematics, vol. 1, no. 3, pp. 309-317, 2012.

[5] A. Mohammed and M. Darus, "Starlikeness properties of a new integral operator for meromorphic functions," Journal of Applied Mathematics, vol. 2011, Article ID 804150, 8 pages, 2011.

[6] A. Soni and S. Kant, "A new subclass of meromorphic closeto-convex functions," Journal of Complex Analysis, vol. 2013, Article ID 629394, 5 pages, 2013.

[7] Q.-H. Xu, H. M. Srivastava, and Z. Li, "A certain subclass of analytic and close-to-convex functions," Applied Mathematics Letters, vol. 24, no. 3, pp. 396-401, 2011.

[8] M.-S. Liu, "A subclass of $p$-valent close-to-convex functions of type $\beta$ and order $\alpha$," Journal of Mathematical Study, vol. 30, no. 1, pp. 102-104, 1997.

[9] Ch. Pommerenke, "On meromorphic starlike functions," Pacific Journal of Mathematics, vol. 13, pp. 221-235, 1963.

[10] A. W. Goodman, Univalent Functions, Mariner, Tampa, Fla, USA, 1983.

[11] J. Clunie, "On meromorphic schlicht functions," Journal of the London Mathematical Society, vol. 34, pp. 215-216, 1959.

[12] W. Rogosinski, "On the coefficients of subordinate functions," Proceedings of the London Mathematical Society, vol. 48, pp. 4882, 1943. 


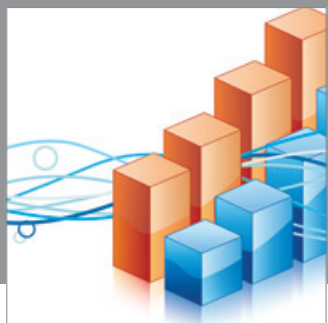

Advances in

Operations Research

mansans

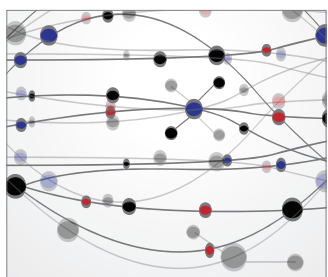

The Scientific World Journal
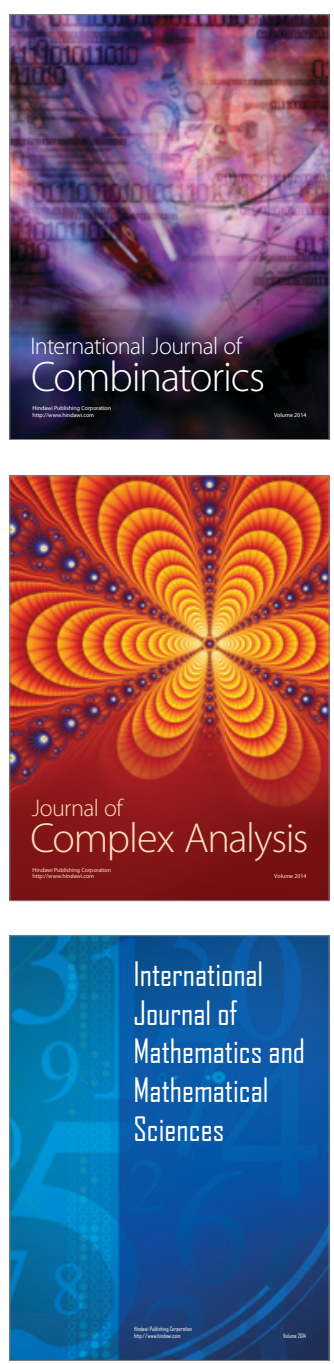
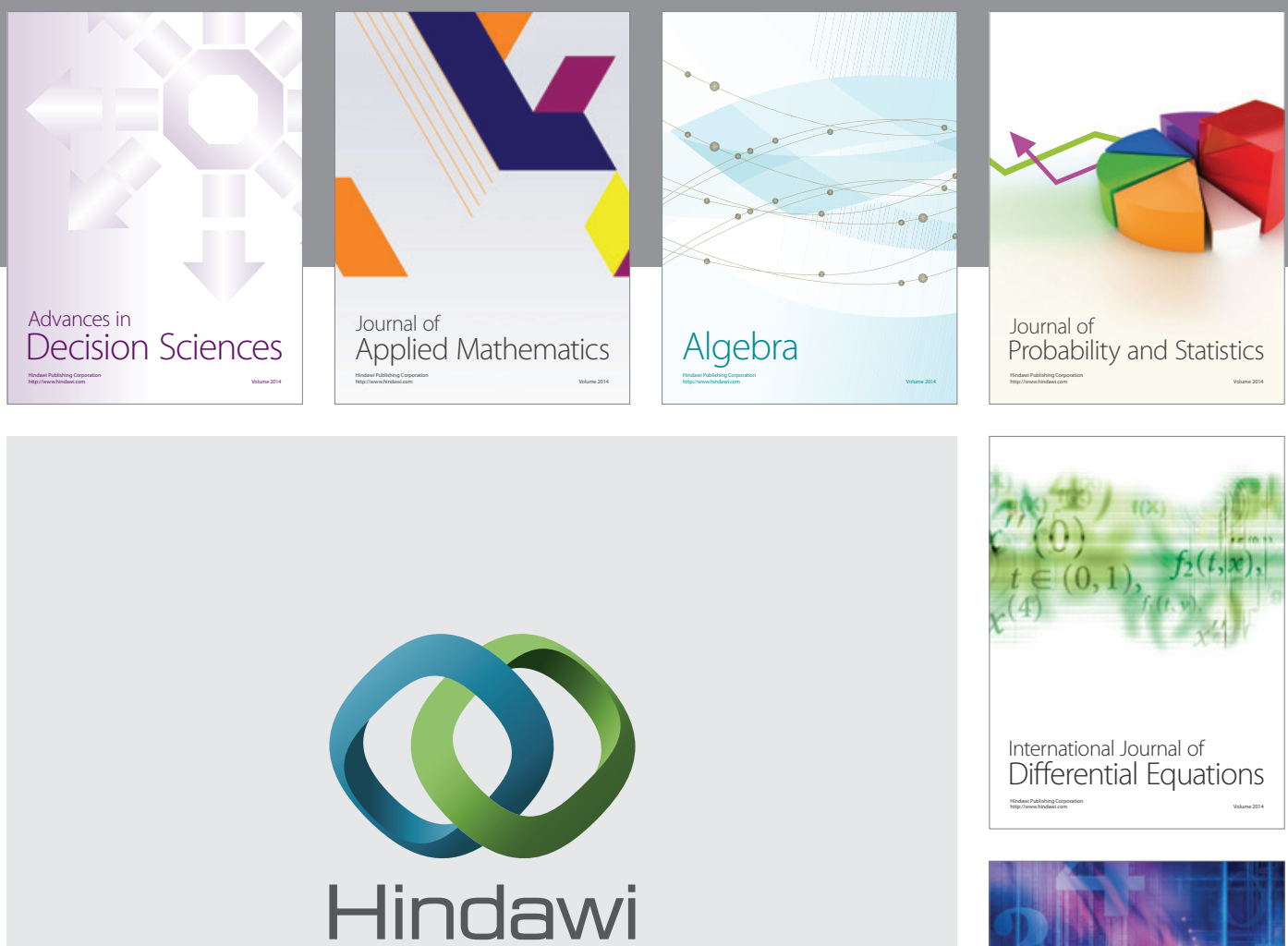

Submit your manuscripts at http://www.hindawi.com
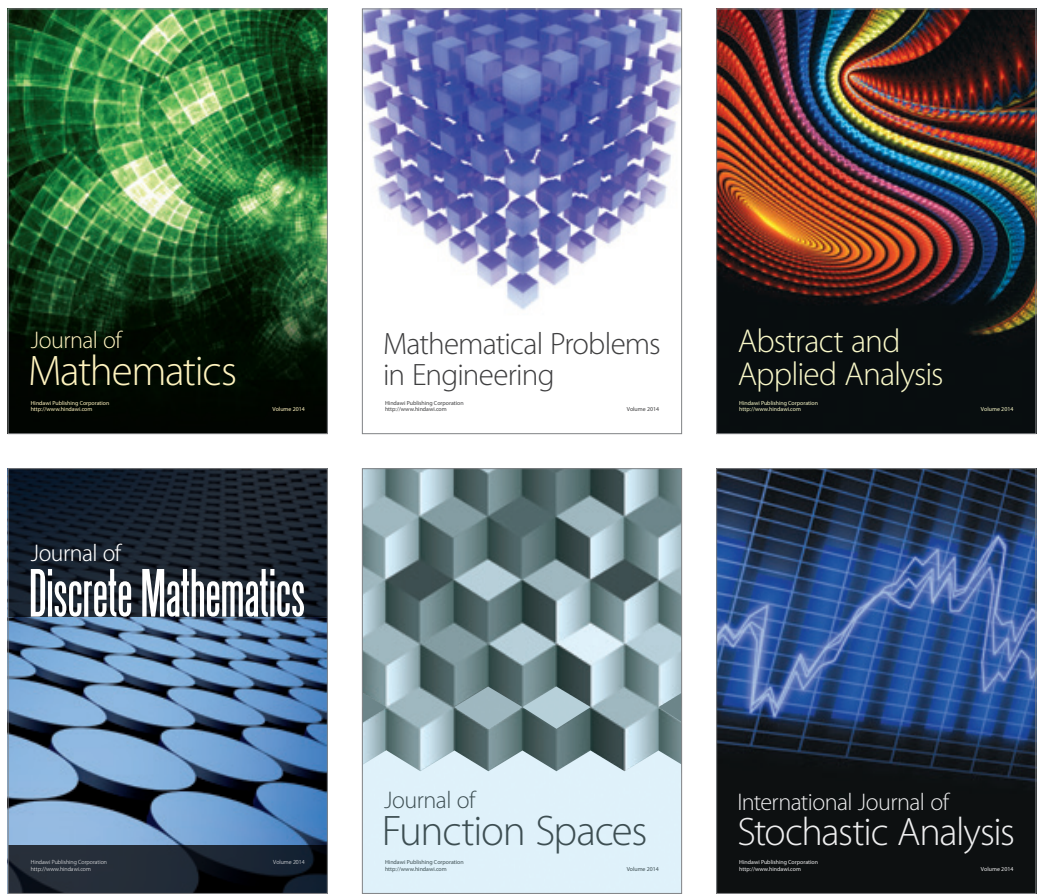

Journal of

Function Spaces

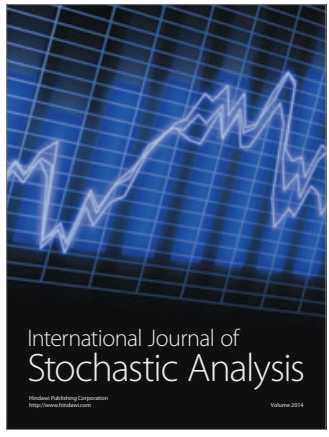

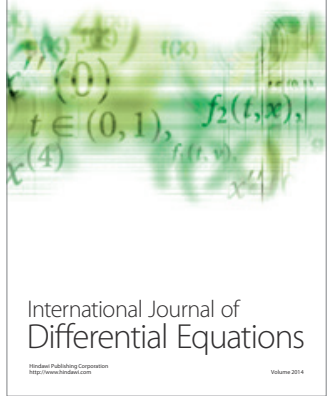
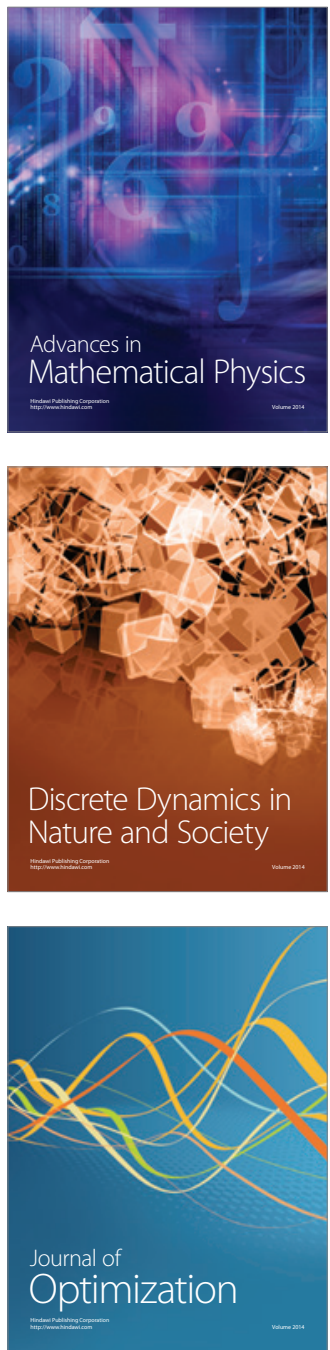